\title{
Analytic expressions for the constitutive parameters of magnetoelectric metamaterials
}

\author{
D. R. Smith* \\ Center for Metamaterials and Integrated Plasmonics and Department of Electrical and Computer Engineering, Duke University, \\ P.O. Box 90291, Durham, North Carolina 27708, USA
}

(Received 23 August 2009; revised manuscript received 21 January 2010; published 17 March 2010)

\begin{abstract}
Electromagnetic metamaterials are artificially structured media typically composed of arrays of resonant electromagnetic circuits, the dimension and spacing of which are considerably smaller than the free-space wavelengths of operation. The constitutive parameters for metamaterials, which can be obtained using fullwave simulations in conjunction with numerical retrieval algorithms, exhibit artifacts related to the finite size of the metamaterial cell relative to the wavelength. Liu et al. [R. Liu, T. J. Cui, D. Huang, B. Zhao, and D. R. Smith, Phys. Rev. E 76, 026606 (2007)] showed that the complicated, frequency-dependent forms of the constitutive parameters can be described by a set of relatively simple analytical expressions. These expressions provide useful insight and can serve as the basis for more intelligent interpolation or optimization schemes. Here, we show that the same analytical expressions can be obtained using a transfer-matrix formalism applied to a one-dimensional periodic array of thin, resonant, dielectric, or magnetic sheets. The transfer-matrix formalism breaks down, however, when both electric and magnetic responses are present in the same unit cell, as it neglects the magnetoelectric coupling between unit cells [C. R. Simovski, Metamaterials 1, 62 (2007)]. We show that an alternative analytical approach based on the same physical model must be applied for such structures. Furthermore, in addition to the intercell coupling, electric and magnetic resonators within a unit cell may also exhibit magnetoelectric coupling. For such cells, we find an analytical expression for the effective index, which displays markedly characteristic dispersion features that depend on the strength of the coupling coefficient. We illustrate the applicability of the derived expressions by comparing to full-wave simulations on magnetoelectric unit cells. We conclude that the design of metamaterials with tailored simultaneous electric and magnetic response-such as negative index materials—will generally be complicated by potentially unwanted magnetoelectric coupling.
\end{abstract}

DOI: 10.1103/PhysRevE.81.036605

PACS number(s): 41.20.Jb, 42.25.Bs, 78.20.-e

\section{INTRODUCTION}

A variety of numerically based retrieval approaches have been developed over the past decade for the purpose of designing and characterizing periodic arrays of scattering elements in terms of effective electromagnetic media [1-6]. The goal of these methods is to remove the details associated with the local field and current distributions that exist in and around the individual elements by the application of an averaging or homogenization scheme. Such homogenization schemes aim to replace the local description of a collection of elements with averaged values for the effective electric permittivity, $\varepsilon_{\text {eff }}$, and effective magnetic permeability, $\mu_{\text {eff }}$. Wave propagation within the effective medium defined by the collection of elements can then be fully described using the derived effective constitutive parameters in conjunction with Maxwell's equations. The use of effective constitutive parameters provides a convenient means to understand wave propagation phenomena in artificially structured metamaterials without the need to consider the complex quasistatic local-field structure around the metamaterial elements.

The process of averaging the fields over a collection of elements to arrive at a continuous medium description is a fundamental procedure that is well established [7]. Analytic expressions have been derived that describe effective media with varying degrees of internal complexity, from simple

\footnotetext{
*drsmith@ee.duke.edu
}

mixtures of dielectrics to materials with much more elaborate inclusions [8-11]. As the field of metamaterials has progressed, however, the need for extremely accurate models of the effective-medium properties of complex scattering elements has also advanced. The approximate analytical constitutive parameters derived for effective media comprising conducting circuit elements $[12,13]$, for example, provide an intuitive, qualitative understanding of elements such as splitring resonator (SRR) or wire-based metamaterials, but are inadequate for precise design. As an alternative approach, numerical retrievals are now routinely applied to unit cells containing arbitrary collections of metamaterial elements. Because periodic boundary conditions can be applied in fullwave simulations, the effective interactions among all of the elements are correctly accounted for. From either the computed local fields or the computed transmission and reflection coefficients, exact values for the effective constitutive parameters can thus be obtained. The numerical approaches have been successful in facilitating the design of exotic media, including metamaterials with negative refractive index [14-20], gradient index metamaterials [21-23], and transformation optical media $[24,25]$.

To obtain the often exotic response that has come to be associated with metamaterials, resonant conducting elements are typically used. These resonant circuits exhibit a dipolar response that can be quite large and either in-phase or outof-phase with the incident driving electric or magnetic field. Although quasistatic models of such metamaterials predict Drude-Lorentz-like resonant effective constitutive parameters $[12,13]$, the actual simulated elements result in far more 
complex (and seemingly unphysical) line shapes that appear difficult to describe in a simple manner. The specific, frequency-dependent form of metamaterial constitutive parameters has been suggested to be a consequence of spatial dispersion $[3,4,26,27]$ - that is, the finite size of the unit cell relative to the wavelength.

In 2007, Liu et al. examined the field-averaging approach to the homogenization of metamaterials and arrived at relatively uncomplicated analytical expressions that could account for the unusual line shapes associated with metamaterial elements [5]. The resulting expressions included a term corresponding to the averaged permittivity (or permeability) - assumed to have a Drude-Lorentz-like form-multiplied by a term accounting for spatial dispersion. The complex line shapes typically obtained by direct numerical simulation, then, could apparently be understood by an analytical expression having only a few free parameters, such as oscillator strength, resonant frequency, damping factor, and unit cell size. It was shown that with the appropriate choice of these constants, a nearly exact match of the analytically versus numerically determined frequencydependent constitutive parameters could be found.

Although not a substitute for numerical simulations, the use of simple expressions to describe the effective constitutive parameters of metamaterials provides considerable convenience and may be particularly useful in the development of intelligent interpolation and optimization schemes. The design of gradient index or transformation optical media, for example, typically requires repetitive simulations in which one or more geometrical parameters of a metamaterial element are altered to produce a range of permittivity or permeability values. The use of an analytic expression can potentially accelerate the various design steps.

The simplicity of the expressions found by Liu et al. [5] is compelling, as is their agreement with numerical simulations. The very simplicity of these expressions in itself suggests that the underlying physical model should also be relatively uncomplicated. We take as an initial goal here to show that the analytical expressions can be, in fact, found as limiting approximations from a picture in which the metamaterial consists of a one-dimensional periodic array of polarizable sheets. A complete and rigorous treatment of the problem, starting from three-dimensional arrays of polarizable dipoles, has been presented by Simovski [28].

A key question that has emerged in parallel with the expanding field of metamaterials concerns the conditions under which a particular retrieval method can be used to ascribe the effective-medium properties of a large or infinite array of scatterers to the properties of a single layer. Simovski defines the concept of a Bloch lattice, in which the sheets interact with each other only via the lowest-order Bloch wave, and the polarizability of each cell is not changed by the presence of the other layers [28]. Under these conditions, which apply to lattices with either predominantly electric or predominantly magnetic response, a transfer-matrix model can be used to arrive at analytical expressions that accurately describe the infinite lattice constitutive parameters. In Sec. III, we illustrate the derivation of these approximate formulas from the analytical transfer-matrix model. If the unit cell can be modeled as a thin sheet that is simultaneously electrically and magnetically polarizable, however, then a nonlocal magnetoelectric coupling occurs and the properties of the single unit cell can be strongly modified by the presence of the neighboring sheets. Under this circumstance, the transfermatrix approach fails to account for the coupling and yields unreliable or erroneous results for the bulk metamaterial. In this case, an analytical expression can be found for the propagation constant by an alternative approach $[6,28]$, which we present in Sec. IV.

The assumption of an infinitely thin magnetoelectric sheet carries the implication that the electric and magnetic dipoles within the same plane do not interact. However, a model in which the unit cell consists of displaced electric and magnetic sheets implies the electric and magnetic dipoles within the unit cell will be coupled. We derive a form for the propagation constant in the presence of such coupling in Sec. V. In Sec. VI, we provide illustrative examples comparing the various analytical expressions to numerical results.

It is important to emphasize that metamaterials do not homogenize in the manner of classical mixtures or composites, but rather occupy a more nebulous realm between homogenized materials and electromagnetic crystalsstructures designed to operate in the regime where the wavelength is on the order of the periodicity of the lattice. The unifying feature of all three systems is that a unique effective refractive index $n$ can typically be found, so, for example, ray optics can be applied where propagating solutions are allowed. What distinguishes homogenized materials from electromagnetic crystals is that a well-defined wave impedance $Z$ can be defined for the former, while the latter is characterized by a Bloch impedance $Z_{B}$ that varies spatially across the unit cell. It is important to note that the retrieved parameters for metamaterials are based on the spatially dispersive Bloch impedance rather than the wave impedance [28], so that $\varepsilon_{B}=n / Z_{B}$ and $\mu_{B}=n Z_{B}$, and will generally be ill-defined at points within the unit cell. Thus, although they can be used to conveniently describe the scattering from metamaterial structures as well as other associated complex behavior, the numerically determined constitutive parameters have limits of applicability. To make clear this distinction, we adopt the terminology of Bloch constitutive parameters throughout this work when referring to the commonly applied metamaterial parameters.

\section{TRANSFER-MATRIX MODEL}

Many numerical techniques based on field averaging and other retrieval methods have been developed to aid in the design and analysis of metamaterial structures. It is a worthwhile endeavor, however, to seek whether the results from full-wave simulations of the exact geometry can be obtained by replacing the specific structures-SRRs, wires, and so forth-by an array of polarizable electric or magnetic dipoles. The advantage of analyzing the dipolar array is that self-consistent, exact, semianalytical expressions can be obtained that inherently account for the effects of spatial dispersion and can provide further insight as to the fundamental intercell and intracell electromagnetic interactions.

The polarization of an infinite array of dipoles under application of a static magnetic or electric field can be com- 


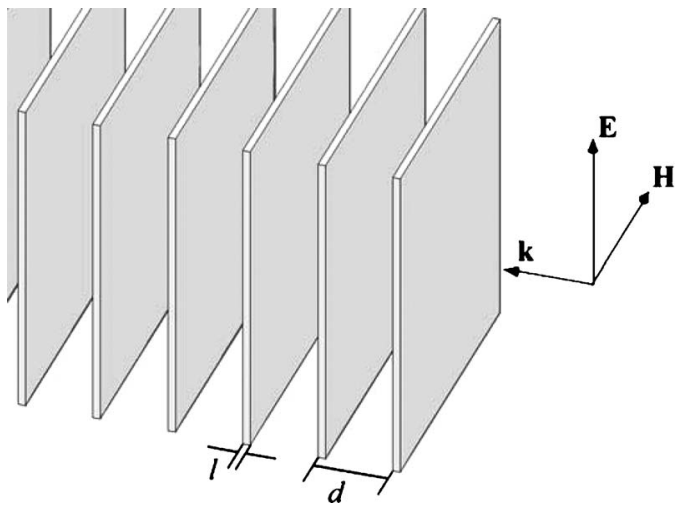

FIG. 1. Periodic system of electrically or magnetically polarizable sheets. The sheets have thickness $l$ and are spaced a distance $d$ apart. An electromagnetic wave is assumed to propagate along the direction normal to the sheets.

puted self-consistently by considering the field at the location of one dipole formed from the summation of the responding fields from all dipoles excluding the given dipole [29]. The effective permittivity or permeability of the collective can then be expressed in terms of the polarizability of the individual dipolar element. For field excitations at finite frequency and wavelength, retardation effects lead to dispersion effects that can be included in the dipole model [30].

Within the scope of the dipole model, it is convenient to first sum over planes of dipoles for periodic arrays and subsequently to take into account the interaction of the planes along the propagation direction $[30,31]$. Since the applied electromagnetic field is uniform over a plane of dipoles (assuming the wave is incident along a principal axis), the effects of spatial dispersion occur due to the phase variation of the field along the direction perpendicular to the planes. Under these conditions, both the effective refractive index and the impedance exhibit spatial dispersion and can explain the structure apparent in the retrieved constitutive parameters for metamaterials [6]. As indicated in Sec. I, we refer to the impedance for such structures as the Bloch impedance.

The unified model followed in this paper will be to consider a wave propagating in an infinite, periodic array of thin, polarizable sheets. Although metamaterial elements certainly have considerable physical extent along the propagation direction within the unit cell, we assume here that their effective dipolar response is restricted to a plane. Though this is a reasonable assertion based on the reflection symmetry of the unit cell, the overall validity of this assumption must be justified by the results given that the wave vector is finite.

The geometry of the model is shown in Fig. 1. A series of polarizable, planar sheets, of width $l$, is spaced apart with periodicity $d$. We assume that an electromagnetic wave propagates in the direction along the normal to the sheets, with fields polarized in the plane of the sheets. The wavepropagation behavior under these assumptions reduces to a one-dimensional boundary-value problem, in which solutions to the wave equation in three regions must be considered: $-d / 2 \leq z<-l / 2,-l / 2 \leq z<l / 2$, and $l / 2 \leq z \leq d / 2$. The dispersion relation and fields for the one-dimensional periodic system can be determined through the use of the transfer-matrix technique [32].
Following the notation used in [3], we present the onedimensional (1D) transfer matrix, which relates the fields on one side of a planar slab to the other. The transfer matrix can be defined from

$$
\mathbf{F}^{\prime}=\mathbf{T F},
$$

where

$$
\mathbf{F}=\left(\begin{array}{c}
E \\
H_{\text {red }}
\end{array}\right)
$$

$E$ and $H_{\text {red }}$ are the complex electric and magnetic field amplitudes located on the right-hand (unprimed) and lefthand (primed) faces of the slab. The magnetic field here is a reduced magnetic field [33] having the normalization $H_{\text {red }}=+i \omega \mu_{0} H$. The transfer matrix for a homogeneous 1D slab has the analytic form

$$
\mathbf{T}=\left(\begin{array}{cc}
\cos (n k d) & -\frac{z}{k} \sin (n k d) \\
\frac{k}{z} \sin (n k d) & \cos (n k d)
\end{array}\right),
$$

where $n$ is the refractive index and $z$ is the wave impedance of the slab. Note that $n$ and $z$ are related to the local relative permittivity and permeability in the usual manner

$$
n=\sqrt{\varepsilon \mu} \quad z=\sqrt{\mu / \varepsilon} .
$$

The fields on the two sides of a unit cell that is composed of three distinct planar regions-vacuum/material/vacuumcan be related by a transfer matrix that consists of the matrix product of the transfer matrices in each region or

$$
\mathbf{T}_{\text {tot }}=\mathbf{T}_{\text {vacuum }} \mathbf{T}_{\text {material }} \mathbf{T}_{\text {vacuum }},
$$

where

$$
\mathbf{T}_{\text {vacuum }}=\left(\begin{array}{cc}
\cos \left(\frac{k d}{2}\right) & -\frac{1}{k} \sin \left(\frac{k d}{2}\right) \\
k \sin \left(\frac{k d}{2}\right) & \cos \left(\frac{k d}{2}\right)
\end{array}\right)
$$

and

$$
\mathbf{T}_{\text {material }}=\left(\begin{array}{lc}
\cos (n k l) & -\frac{z}{k} \sin (n k l) \\
\frac{k}{z} \sin (n k l) & \cos (n k l)
\end{array}\right) .
$$

Using the transfer-matrix formalism with periodic boundary conditions [3], we arrive at the following expressions for the propagation factor and Bloch impedance:

$$
2 \cos (\alpha d)=T_{11}+T_{22}
$$

and

$$
Z_{B, \text { red }}^{2}=\frac{T_{12}}{T_{21}},
$$

where the latter expression is true provided the unit cell possesses reflection symmetry in the direction of propagation. 
Even though this assumption of symmetry may not hold exactly throughout this analysis, we assume Eq. (9) holds approximately. The constitutive parameters determined by using Eqs. (8) and (9) with Eq. (4) are termed the Bloch constitutive parameters [28]. These material parameters are nonlocal; though they have proven a nearly indispensable tool for metamaterial design, they nevertheless do not represent classical homogenization parameters. Because our goal is to develop an understanding of the numerical simulation results, we restrict our focus here to the comparison of analytically and numerically derived Bloch constitutive parameters.

Note that in the static limit, the effective-medium parameters should represent simple volume averages over the dielectric and magnetic regions in the unit cell. That is, for the static limit, we expect that

$$
\begin{aligned}
& \bar{\varepsilon}=\frac{d+\varepsilon l}{d+l} \sim \frac{d+\varepsilon l}{d}, \\
& \bar{\mu}=\frac{d+\mu l}{d+l} \sim \frac{d+\mu l}{d},
\end{aligned}
$$

where we have made the assumption that the thickness of the magnetodielectric sheet is negligible. We have also assumed the absence of magnetoelectric coupling, which we will reexamine in a later section.

\section{ELECTRIC OR MAGNETIC LATTICES}

Since our goal is to find relatively simple analytic expressions for the constitutive parameters in the presence of spatial dispersion, we apply several approximations that we expect to be consistent with resonant metamaterials. In particular, since the unit cell of the metamaterial is relatively small with respect to the wavelength, we expect that $k d$ should be small; that is, most of the phase shifts associated with the propagating wave will occur across the polarizable sheet. Since we are considering very thin sheets, we take the limit $l \rightarrow 0$, yet retain a finite-dipole moment for the thin sheet. We must then require that the electric permittivity or magnetic permeability (but not both) of the sheet tends to infinity. For the case that the sheet has a predominantly electric polarizability, we require

$$
\varepsilon l \rightarrow \text { const, } \quad \sqrt{\varepsilon} l \rightarrow 0 .
$$

A similar approximation was employed in [34] to compute the reflection and transmission properties of a thin sheet of material. Applying this approximation to Eq. (5), we obtain

$$
\mathbf{T}_{e}=\mathbf{T}_{\text {vacuum }}\left(\begin{array}{cc}
1 & 0 \\
\frac{k q l}{z} & 1
\end{array}\right) \mathbf{T}_{\text {vacuum }}
$$

Multiplying out the matrix elements and applying Eq. (8), we arrive at the dispersion relation

$$
\cos \alpha d=\cos k d-\frac{\varepsilon k l}{2} \sin k d,
$$

which relates the phase advance $\alpha d$ across the cell to the properties of the sheet and the unit cell dimension. Equation (13) is identical to the dispersion relation derived for transmission lines with periodic shunt impedance loads [31]. If we now assume that the phase advance in the vacuum region is small, we can write

$$
\cos \alpha d=1-\frac{(k d)^{2}}{2}-\varepsilon l \frac{k d}{2}
$$

or, equivalently,

$$
\sin ^{2} \frac{\alpha d}{2}=\left(\frac{k d}{2}\right)^{2}\left(\frac{d+\varepsilon l}{d}\right) .
$$

Using Eq. (10), we arrive at the simple expression

$$
\sin \frac{\alpha d}{2}=\sqrt{\bar{\varepsilon}} \frac{k d}{2} .
$$

This form of the dispersion relation is consistent with that obtained by field averaging homogenization methods previously reported [4]. Note that it is not possible to simplify the expression further since we cannot assume the Bloch phase advance to be small. Since the relationship between $\alpha$ and $k(=\omega / c)$ represents the dispersion relation for the periodic system, it is reasonable to introduce an effective refractive index defined as

$$
\alpha=n_{\text {eff }} \frac{\omega}{c}=\sqrt{\varepsilon_{B} \mu_{B}} k,
$$

where we have related the effective index to the Bloch constitutive parameters. The average constitutive parameter $\bar{\varepsilon}$ is not bounded (it can be described by a Drude-Lorentz-like resonance, for example), so that the influence of spatial dispersion introduces artifacts into the effective index according to

$$
\alpha=\frac{2}{d} \sin ^{-1} \sqrt{\frac{\bar{\varepsilon}}{\varepsilon}} \frac{k d}{2}=n_{e f f} k
$$

by virtue of the bounded nature of the sine function. A specific difference between a continuous medium and the metamaterial is the existence of a band-gap or staggered mode region in the latter where the magnitude of the argument of the arcsine function exceeds unity, as has been previously pointed out $[5,28]$. The presence of spatial dispersion no longer allows us to simply assume that there is only a Bloch permittivity, so we include the possibility that the Bloch permeability, $\mu_{B}$, has some value distinct from unity even though the average permeability has been assumed as $\bar{\mu}=1$.

Much of the phenomena associated with metamaterials result from the use of structures that exhibit resonant permittivity or permeability. To illustrate the behavior of such resonant media, we consider example cases in which the slabs possess Lorentzian-like constitutive parameters or 


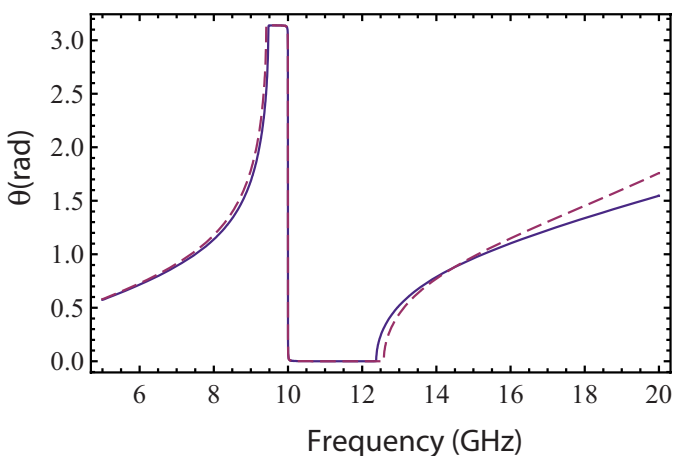

FIG. 2. (Color online) Phase advance $(\theta=\alpha d)$ as a function of frequency for a medium comprising electrically resonant slabs separated by regions of free space. Solid line corresponds to the exact dispersion relation of the slab model, Eq. (13), while dashed line corresponds to the approximate expression found in Eq. (16).

$$
\begin{gathered}
\varepsilon(\omega)=\varepsilon_{b}-\frac{f_{p e}^{2}}{f^{2}-f_{0 e}^{2}+i \Gamma_{e} f}, \\
\mu(\omega)=\mu_{b}-\frac{f_{p m}^{2}}{f^{2}-f_{0 m}^{2}+i \Gamma_{m} f} .
\end{gathered}
$$

Consider a medium for which the relative permeability of the slab is equal to unity and the permittivity has the resonant form in Eq. (19), with $f_{p e}=35 \mathrm{GHz}, f_{0 e}=10 \mathrm{GHz}$, and $\Gamma_{e}$ $=0.001 \mathrm{GHz}$. The unit cell size is $d=0.4 \mathrm{~cm}$ while the slab thickness is $l=0.02 \mathrm{~cm}$. The phase advance per unit cell, $\theta$ $=\alpha d$, is plotted in Fig. 2 as a function of frequency using both the exact expression in Eq. (13) and the approximate Eq. (16). Because the parameters have been chosen specifically to ensure that $k d<1$, we see that Eqs. (13) and (16) agree quite well. The agreement is not general, however, and worsens as $k d$ becomes larger.

To obtain explicit expressions for the effective permittivity or permeability, it is necessary to find an expression for the Bloch impedance. Using Eq. (9) with the matrix elements in Eq. (12) leads to

$$
Z_{B, \text { red }}^{2}=-\frac{1}{k^{2}} \frac{\tan ^{2} \frac{k d}{2}}{\tan ^{2} \frac{\alpha d}{2}}
$$

after applying Eq. (13) and with some further algebraic manipulations. This expression for the impedance is consistent with that found from transmission line models [31]. Since $H_{\text {red }}=+i \omega \mu_{0} H$ and assuming small $k d$ as before, we find

$$
Z_{B}=\sqrt{\frac{\mu_{B}}{\varepsilon_{B}}}=\frac{\frac{k d}{2} \cos \frac{\alpha d}{2}}{\sin \frac{\alpha d}{2}},
$$

which, after substituting in Eq. (16), yields

$$
Z_{B}=\sqrt{\frac{\mu_{B}}{\varepsilon_{B}}}=\frac{\cos \frac{\alpha d}{2}}{\sqrt{\bar{\varepsilon}}} .
$$

Equation (22) reveals that the wave impedance exhibits spatial dispersion in the same form as was found in [5]. Combining Eqs. (17) and (22), we arrive at the simple expression

$$
n_{e f f}=\sqrt{\varepsilon_{B} \mu_{B}}=\sqrt{\bar{\varepsilon}} \frac{\frac{\alpha d}{2}}{\sin \frac{\alpha d}{2}}
$$

for the effective index. From Eqs. (22) and (23), we thus find directly the approximate expressions for the Bloch material parameters

$$
\begin{gathered}
\varepsilon_{B}=\bar{\varepsilon} \frac{\frac{\alpha d}{2}}{\sin \frac{\alpha d}{2} \cos \frac{\alpha d}{2}}, \\
\mu_{B}=\frac{\frac{\alpha d}{2}}{\sin \frac{\alpha d}{2} \cos \frac{\alpha d}{2},},
\end{gathered}
$$

in agreement with [5]. We see that the Bloch permittivity is simply the averaged permittivity multiplied by a term that depends only on the phase advance across the cell, $\alpha d$. And, though there is no inherent averaged magnetic response, the effective permeability is no longer equal to unity but will exhibit structure due to the effects of the spatial dispersion terms.

If, instead of an electric response the thin sheet has primarily a magnetic response, then we can take a similar limit as in Eq. (11), requiring

$$
\mu l \rightarrow \text { const }, \quad \sqrt{\mu} l \rightarrow 0 .
$$

In this limit, the transfer matrix becomes

$$
\mathbf{T}_{m}=\mathbf{T}_{\text {vacuum }}\left(\begin{array}{cc}
1 & -\frac{z q l}{k} \\
0 & 1
\end{array}\right) \mathbf{T}_{\text {vacuum }} .
$$

Applying Eq. (8), as before, we arrive at the expression

$$
\cos \alpha d=\cos k d-\frac{\mu k l}{2} \sin k d .
$$

Again, under the assumption that the phase advance in the vacuum region is small, we can expand the trigonometric functions to obtain

$$
\sin \frac{\alpha d}{2}=\sqrt{\bar{\mu}} \frac{k d}{2} .
$$

Likewise, following the same steps as outlined above, we find the reduced impedance for the magnetic lattice is 


$$
Z_{B, \text { red }}^{2}=-\frac{1}{k^{2}} \frac{\tan ^{2}\left(\frac{\alpha d}{2}\right)}{\tan ^{2} \frac{k d}{2}} .
$$

Using Eq. (29) with Eq. (27) yields

$$
Z_{B}=\sqrt{\frac{\mu_{B}}{\varepsilon_{B}}}=\sqrt{\bar{\mu}} \frac{1}{\cos \frac{\alpha d}{2}},
$$

which, when following the steps that led to Eq. (23), provides the Bloch constitutive parameters as

$$
\begin{gathered}
\varepsilon_{B}=\frac{\frac{\alpha d}{2}}{\sin \frac{\alpha d}{2}} \cos \frac{\alpha d}{2}, \\
\mu_{B}=\bar{\mu} \frac{\frac{\alpha d}{2}}{\sin \frac{\alpha d}{2} \cos \frac{\alpha d}{2}} .
\end{gathered}
$$

Equations (24) and (31) provide a useful description of metamaterials having either predominantly electric or predominantly magnetic response, respectively. These expressions will be compared to numerical retrievals in Sec. VI.

\section{MAGNETOELECTRIC LATTICES}

Although it would be appealing to consider the same approach as above to analyze lattices composed of magnetodielectric sheets, the use of the transfer-matrix method, in fact, fails for such cases due to the inherent neglect of magnetoelectric interactions between the planes $[6,32,33]$. Were we to follow the transfer-matrix approach, we might approximate the electric and magnetic dipolar arrays as two thin sheets: one electrically polarizable and the other magnetically polarizable. The transfer matrix for the unit cell would then be

$$
\mathbf{T}_{e m}=\mathbf{T}_{\text {vacuum }}\left(\begin{array}{cc}
1 & 0 \\
\frac{k q l}{z} & 1
\end{array}\right)\left(\begin{array}{cc}
1 & -\frac{z q l}{k} \\
0 & 1
\end{array}\right) \mathbf{T}_{\text {vacuum }} .
$$

The dispersion relation could then be found numerically using Eq. (8) above or from the approximate expression

$$
\sin \frac{\alpha d}{2}=\sqrt{\bar{\varepsilon} \bar{\mu}} \frac{k d}{2} .
$$

Unfortunately, this expression is not correct because it neglects the magnetoelectric coupling between the electric and magnetic layers. Moreover, most metamaterial unit cells that contain magnetic and electric components will exhibit magnetoelectric coupling between the components within the unit cell, thus making the assumption of only a permittivity and a permeability erroneous from the outset. Still, it is of interest to see if an analytical expression can be obtained for the propagation constant of a magnetoelectric lattice similar to Eq. (33).

To account for the magnetoelectric coupling between unit cells, we follow the approach of Simovski [28] to derive the dispersion relation directly for the magnetoelectric slab system and deduce approximate results subsequently. Because point electric and magnetic dipoles lying within the same plane do not exhibit magnetoelectric coupling, we consider first the hypothetical case of an infinite medium composed of infinitely thin sheets that are simultaneously electrically and magnetically polarizable.

The geometry is the same as in Fig. 1, except that the planes now possess both magnetic and electric responses in the form of a permeability and permittivity. If we first consider just the electric response, we imagine that a displacement current density is formed in the slab at $x=0$ in proportion to the local electric field. That is,

$$
J_{e}=\frac{\partial D}{\partial t}=-i \omega \varepsilon E_{l o c} .
$$

Because we expect the slabs to be very thin, so that the thickness $l$ is negligible, we treat the responding current as a surface current $K_{e}$ or

$$
J_{e}=K_{e} / l
$$

We thus can define an effective, local response function according to

$$
K_{e}=-i \omega \varepsilon l E_{l o c}=\alpha_{e} E_{l o c} .
$$

A uniform sheet of current located at $x=0$ will launch a one-dimensional wave having a form that can be determined from the boundary conditions. Using the curl equation, we find the magnitude of the launched wave as

$$
H=\frac{K_{e}}{2} .
$$

Given that the wave propagates in free space, the electric field is easily found as $E=\eta H=\eta K_{e} / 2$ or

$$
E(x)=\frac{\eta}{2} \alpha_{e} e^{i k|x|},
$$

where $\eta=\sqrt{\mu_{0} / \varepsilon_{0}}$. The interaction of the slab at $x=0$ with the infinite lattice can be found by summing over the field produced at $x=0$ from the responding currents in each of the other slabs. Given that the system is periodic, the responding surface currents must bear the following phase relationship with respect to one another:

$$
K_{e, n}=K_{e, 0} e^{i n \alpha d},
$$

where $n= \pm 1, \pm 2, \pm 3, \ldots$ The total field, then, acting at $x$ $=0$ will be 


$$
E(0)=\sum_{n} \frac{\eta}{2} e^{i k|n| d} e^{i n \alpha d} K_{e, 0}
$$

The summation can be performed using standard methods and we arrive at the following expression:

$$
E(0)=\frac{\eta}{2}\left[\frac{-i \sin k d}{\cos k d-\cos \alpha d}\right] K_{e, 0} .
$$

In the absence of any magnetic response, we can utilize Eq. (41) with Eq. (38) to obtain the dispersion relation

$$
\cos \alpha d=\cos k d-i \frac{\eta \alpha_{e}}{2} \sin k d
$$

which is identical to Eq. (13) above. In general, however, if there is also a magnetic response present, then it is necessary to account for a separate, nonlocal magnetoelectric coupling. We can define a magnetic sheet current, analogous to Eq. (36), as

$$
K_{m}=-i \omega \mu l H_{l o c}=\alpha_{m} H_{l o c} .
$$

Because the electric current launches an electromagnetic wave, the wave will excite magnetic currents in the magnetoelectric slabs, producing a net magnetic field back at the origin. Following the same approach as above, we can write that the magnetic field produced by the electric current in the sheet is

$$
H(x)= \pm \frac{\alpha_{e}}{2} e^{i k|x|},
$$

where the plus or minus sign depends whether the observation position is to the right or the left of the originating sheet current, respectively. This magnetic field will induce magnetic currents in each of the sheets in the array. As before, the periodic boundary conditions require that each sheet retains the phase relationship

$$
K_{m, n}=K_{m, 0} e^{i n \alpha d}
$$

relative to the sheet at $x=0$. We then find, for the magnetic field acting at the origin,

$$
H(0)=\sum_{n \neq 0} \pm \frac{1}{2} e^{i k|n| d} e^{i n \alpha d} K_{e, 0}
$$

or, summing the series as before,

$$
H(0)=\frac{\sin \alpha d}{\cos k d-\cos \alpha d} K_{e, 0} .
$$

Now, given the cross coupling that exists between the electric and magnetic responses in the slab system, the following system of equations must be satisfied:

$$
E=A K_{e}+B K_{m}=\frac{K_{e}}{\alpha_{e}},
$$

$$
H=C K_{e}+D K_{m}=\frac{K_{m}}{\alpha_{m}} .
$$

From reciprocity conditions, it must be true that $A=\eta^{2} D$ and $B=C$ [28]. Thus, setting the determinant of Eq. (48) to zero, we find

$$
\begin{aligned}
\cos \alpha d= & \cos k d-\sin k d\left(\frac{\eta \alpha_{e}}{4}+\frac{\alpha_{m}}{4 \eta}\right) \\
& \pm \sqrt{\sin ^{2} k d\left(\frac{\eta \alpha_{e}}{4}-\frac{\alpha_{m}}{4 \eta}\right)^{2}+\frac{\alpha_{e} \alpha_{m}}{4} \sin ^{2} \alpha d}
\end{aligned}
$$

Though found for a system of magnetodielectric sheets, Eq. (49) nevertheless is identical to the expression found through an analysis of interacting (three-dimensional) magnetic and electric dipoles [6,32,33]. Notably, Eq. (49) is complicated by the inherent nonlocal interaction caused by the excitation of electric and magnetic responses in different planes. A dispersion relation of this form cannot be found via the transfermatrix procedure outline in Sec. II, which treats all responses as local (as pointed out in [33]).

Writing Eq. (49) in terms of the relative permittivity and permeability, we see that

$$
\begin{aligned}
\cos \alpha d= & \cos k d-\sin k d\left(\frac{k \varepsilon l}{4}+\frac{k \mu l}{4}\right) \\
& \pm \sqrt{\sin ^{2} k d\left(\frac{k \varepsilon l}{4}-\frac{k \mu l}{4}\right)^{2}+\frac{\varepsilon \mu k^{2} l^{2}}{4} \sin ^{2} \alpha d} .
\end{aligned}
$$

In general, there are no obvious approximations that should yield generic formulas analogous to Eq. (33). However, we might consider the case that the electric and magnetic responses are large and are roughly of the same order, in which case we can approximate Eq. (50) as

$$
2 \sin ^{2} \frac{\alpha d}{2}=\frac{(k d)^{2}}{2}\left[1+\left(\frac{\varepsilon l}{2 d}+\frac{\mu l}{2 d}\right)\right] \mp \frac{\sqrt{\varepsilon \mu k} l}{2} \sin \alpha d .
$$

If the phase advance across the air portion of the unit cell is small enough, then we can neglect the first term, arriving at the following approximate dispersion relation:

$$
\tan \frac{\alpha d}{2}=\sqrt{\varepsilon \mu\left(\frac{l}{d}\right)^{2}} \frac{k d}{2} \approx \sqrt{\bar{\varepsilon} \bar{\mu}} \frac{k d}{2} .
$$

Equation (52) is remarkably similar to Eq. (33), but a tangent function appears on the left-hand side rather than the sine function. Because the tangent function is not bounded, the band-gap or staggered mode region is suppressed and propagation solutions can exist at all frequencies under the right circumstances. It should be noted, however, that Eq. (52) is highly restricted in its use, corresponding specifically to the case of electric and magnetic resonators that are resonant at the same frequency and with similar dispersion characteristics. 


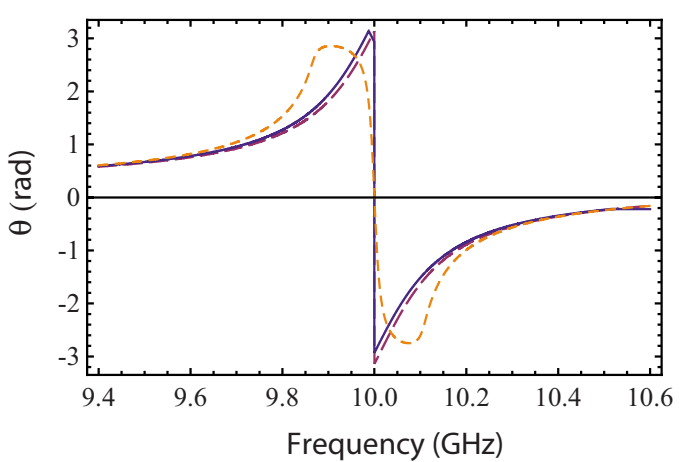

FIG. 3. (Color online) Plot of phase advance $\theta=\alpha d$ as a function of frequency near the resonance of a magnetoelectric structure, consisting of an array of thin slabs having simultaneous resonances in the permittivity and permeability. The resonance frequencies are both at $10 \mathrm{GHz}$, with all other parameters identical. Solid line corresponds to the exact dispersion relation of Eq. (50), while red dashed line corresponds to the approximate dispersion relation in Eq. (52). For the region where $k d<1$, the curves for the two cases are nearly identical. The dispersion relation developed assuming no magnetic electric coupling [Eq. (33)] leads to a curve with very different behavior (short dashed orange curve).

It should also be pointed out that the term "refractive index" is somewhat misleading here, since the magnetoelectric coupling produces a bianisotropic medium. Waves propagating along the selected axis maintain their transverse electric (TE) or transverse magnetic (TM) polarization state; however, waves propagating along arbitrary directions will generally exhibit polarization conversion. Though it is possible to obtain an analytical expression for the wave impedance, as found above, the use of the wave impedance with the effective index defined using Eq. (52) would not lead directly to reasonable expressions for the Bloch constitutive parameters. Thus, for this and the following section, we restrict our attention to the effects of magnetoelectric coupling on the propagation coefficient, which we also define as the effective index.

The phase advance per unit cell for a lattice formed from magnetoelectric sheets is shown in Fig. 3. A magnetoelectric slab was chosen with thickness $l=0.01 \mathrm{~cm}$, with both resonant permittivity and permeability of the form $f_{p e}=f_{p m}$ $=15 \mathrm{GHz}, f_{0 e}=f_{0 m}=10 \mathrm{GHz}$, and $\Gamma_{e}=\Gamma_{m}=0.02 \mathrm{GHz}$. The periodicity of the slabs was $d=0.1 \mathrm{~cm}$. The phase advance per unit cell, $\theta=\alpha d$, plotted in Fig. 3, exhibits considerably different behavior than that corresponding to a medium with either electric or magnetic slabs only. The parameters for Fig. 3 were deliberately chosen such that Eq. (52) would represent a reasonable approximation and that Eq. (52) would show distinct behavior from that of Eq. (33), incorrectly derived excluding the magnetoelectric coupling. The blue dashed line in the figure, corresponding to Eq. (52), shows the tangent form and is in excellent agreement with the exact dispersion relation. The incorrect expression [Eq. (33)], plotted as the orange dashed line, exhibits a band-gap behavior on either side of the resonance.

The typically used approach to retrieve the Bloch constitutive parameters relies on computing the transmission and reflection coefficients for a single unit cell. Thus, the consti-

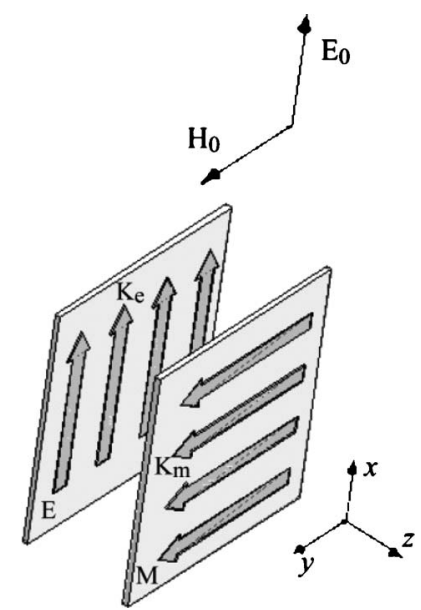

FIG. 4. Unit cell composed of interacting electric and magnetic sheets. Magnetic currents in the magnetic layer produce an electric field that drives currents in the electric layer, while electric currents in the electric layer produce a magnetic field that drives currents in the magnetic layer.

tutive parameters retrieved via this approach are unlikely to agree with those for an actual structure with many unit cells in the propagation direction, in which the cell-to-cell coupling will begin to dominate. In order that the usual retrieval approach be predictive for such structures, it is necessary that the cell-to-cell coupling be weak enough to be neglected. Since this cannot be known for certain in advance, it is likely other numerical techniques will need to be applied during the design phase to assess the degree of magnetoelectric coupling.

If the symmetry of the unit cell is such that magnetoelectric coupling occurs within the unit cell, then it is possible this coupling will dominate and the cell-to-cell coupling may be secondary or negligible in comparison. This circumstance is considered in the next section, with numerical retrieval results provided in Sec. IV.

\section{STRONGLY MAGNETOELECTRIC UNIT CELLS}

In the above analysis, we considered an infinite array of magnetoelectric lattices in which the electric and magnetic responses within the same unit cell are rigorously uncoupled. That is, the electric polarizability of a given sheet is not affected by the magnetic polarizability in the same sheet, though it is affected by the sheets in the rest of the lattice. However, in most unit cell designs, there typically exists an explicit magnetoelectric interaction due to the inherent difficulties associated with producing entirely decoupled resonators. We now consider the impact of magnetoelectric coupling within the unit cell (Fig. 4).

Consider a unit cell containing two polarizable sheets placed next to each other: one electric and the other magnetic. The sheets are placed close enough together that propagation delay between them can be neglected. The electric currents in the electrically polarizable sheet will be proportional to the local electric field. Likewise, the magnetic currents in the magnetically polarizable sheet will be proportional to the local magnetic field or 


$$
\begin{gathered}
K_{e}=\alpha_{e} E_{l o c}, \\
K_{m}=\alpha_{m} H_{l o c} .
\end{gathered}
$$

Assuming the currents are excited by a plane wave with fields $E_{0}$ and $H_{0}$, we can write

$$
\begin{aligned}
& E_{l o c}=E_{0}+\alpha_{e m}^{-1} K_{m}, \\
& H_{l o c}=H_{0}-\alpha_{m e}^{-1} K_{e} .
\end{aligned}
$$

Though we have omitted the indices, it is assumed that the electric field lies along the $x$ direction, while the magnetic field lies along the $y$ direction. Using Eqs. (53) and (54), we can write the following expression for the net response of the system:

$$
\left(\begin{array}{l}
K_{e} \\
K_{m}
\end{array}\right)=\frac{1}{1+\alpha_{e} \alpha_{m} / \alpha_{e m} \alpha_{m e}}\left(\begin{array}{cc}
\alpha_{e} & \frac{\alpha_{e} \alpha_{m}}{\alpha_{e m}} \\
-\frac{\alpha_{e} \alpha_{m}}{\alpha_{m e}} & \alpha_{m}
\end{array}\right)\left(\begin{array}{c}
E_{0} \\
H_{0}
\end{array}\right)
$$

or

$$
\left(\begin{array}{c}
K_{e} \\
K_{m}
\end{array}\right)=\left(\begin{array}{cc}
\alpha_{e, e f f} & \alpha_{e m, e f f} \\
\alpha_{m e, e f f} & \alpha_{m, e f f}
\end{array}\right)\left(\begin{array}{c}
E_{0} \\
H_{0}
\end{array}\right) .
$$

Using Eqs. (36) and (43), we can rewrite Eq. (56) as

$$
\left(\begin{array}{l}
K_{e} \\
K_{m}
\end{array}\right)=\frac{1}{1-\frac{\omega^{2} d^{2} \overline{\varepsilon \mu}}{\alpha_{e m} \alpha_{m e}}}\left(\begin{array}{cc}
-i \omega d \bar{\varepsilon} & -\frac{\omega^{2} d^{2} \overline{\varepsilon \mu}}{\alpha_{e m}} \\
+\frac{\omega^{2} d^{2} \overline{\varepsilon \mu}}{\alpha_{m e}} & -i \omega d \bar{\mu}
\end{array}\right)\left(\begin{array}{l}
E_{0} \\
H_{0}
\end{array}\right)
$$

and define a set of effective constitutive parameters as

$$
\left(\begin{array}{ll}
\varepsilon_{e f f} & \xi_{\text {eff }} \\
\zeta_{\text {eff }} & \mu_{e f f}
\end{array}\right)=\frac{1}{1-\frac{\omega^{2} d^{2} \overline{\varepsilon \mu}}{\alpha_{e m} \alpha_{m e}}}\left(\begin{array}{ccc}
\bar{\varepsilon} & i \omega d \frac{\overline{\varepsilon \mu}}{\alpha_{e m}} \\
-i \omega d \frac{\overline{\varepsilon \mu}}{\alpha_{m e}} & \bar{\mu}
\end{array}\right) .
$$

Because we consider one-dimensional wave propagation in an anisotropic medium, we assume a wave propagating along the $z$ direction, with electric and magnetic fields polarized along the $x$ and $y$ axes, respectively. The Maxwell curl equations can then be written as

$$
\begin{aligned}
& \frac{\partial E_{x}}{\partial z}=i \omega\left(\mu_{e f f} H_{y}+\zeta_{e f f} E_{x}\right), \\
& \frac{\partial H_{y}}{\partial z}=i \omega\left(\xi_{e f f} H_{y}+\varepsilon_{e f f} E_{x}\right),
\end{aligned}
$$

leading to the dispersion relation

$$
\left(i k-i \omega \zeta_{e f f}\right)\left(i k-i \omega \xi_{e f f}\right)+\omega^{2} \varepsilon_{e f f} \mu_{e f f}=0 .
$$

Constraining the medium to be reciprocal and to satisfy timereversal symmetry leads to $\alpha_{m e}=\alpha_{e m} \equiv 2 / i \kappa$. We have chosen the specific form for the magnetoelectric coupling parameter, recognizing that maximal coupling should occur at a minimum value of $\alpha_{m e}=\alpha_{e m}=2$. Equation (58) can then be written as

$$
\left(\begin{array}{ll}
\varepsilon_{e f f} & \xi_{\text {eff }} \\
\zeta_{\text {eff }} & \mu_{\text {eff }}
\end{array}\right)=\frac{1}{1+\omega^{2} d^{2} \overline{\varepsilon \mu}\left(\frac{\kappa}{2}\right)^{2}}\left(\begin{array}{cc}
\bar{\varepsilon} & -\frac{\omega d \overline{\varepsilon \mu} \kappa}{2} \\
\frac{\omega d \overline{\varepsilon \mu} \kappa}{2} & \bar{\mu}
\end{array}\right)
$$

The medium defined by Eq. (61) with $\kappa$ either real or imaginary is bianisotropic and has electromagnetic properties consistent with the symmetry of the unit cell $[34,35]$. Using Eqs. (59)-(61), we are able to write an effective refractive index as

$$
n=\frac{\sqrt{\overline{\varepsilon \mu}}}{\sqrt{1+\overline{\varepsilon \mu}\left(\frac{k d}{2}\right)^{2} \kappa^{2}}} .
$$

Finally, motivated by Eq. (40), we write the following form that includes the effects of the periodic structure:

$$
\sin \left(\frac{\alpha d}{2}\right)=\frac{\sqrt{\overline{\varepsilon \mu}} \frac{k d}{2}}{\sqrt{1+\overline{\varepsilon \mu}\left(\frac{k d}{2}\right)^{2} \kappa^{2}}} .
$$

Equation (63) provides the approximate description of an infinite medium consisting of unit cells with electric and magnetic resonators that are coupled, but neglects the cellto-cell magnetoelectric coupling. If the internal coupling dominates over the cell-to-cell magnetoelectric coupling, then it might be expected that Eq. (63) should be approximately valid. At a minimum, Eq. (63) should be consistent with numerical retrievals performed on a single magnetoelectric unit cell. Comparing Eq. (63) to the incorrect Eq. (33), we see that the forms are similar if we imagine the effective permittivity and permeability are renormalized by the denominator in Eq. (63). In fact, a more detailed analysis shows that the renormalized permittivity and permeability in Eq. (63) are approximately Lorentzian-like, meaning that Eq. (33) could still prove useful for curve fitting if the Lorentzian parameters applied are not required to be consistent with any physical model.

We have written the dispersion relation in a manner such that when the magnetoelectric coupling parameter, $\kappa$, approaches unity, we have

$$
\tan \left(\frac{\alpha d}{2}\right)=\sqrt{\overline{\varepsilon \mu}} \frac{k d}{2},
$$

which is reminiscent of Eq. (52). We illustrate the qualitative effects of a constant coupling parameter on the refractive index profile of a unit cell containing a magnetic and an 


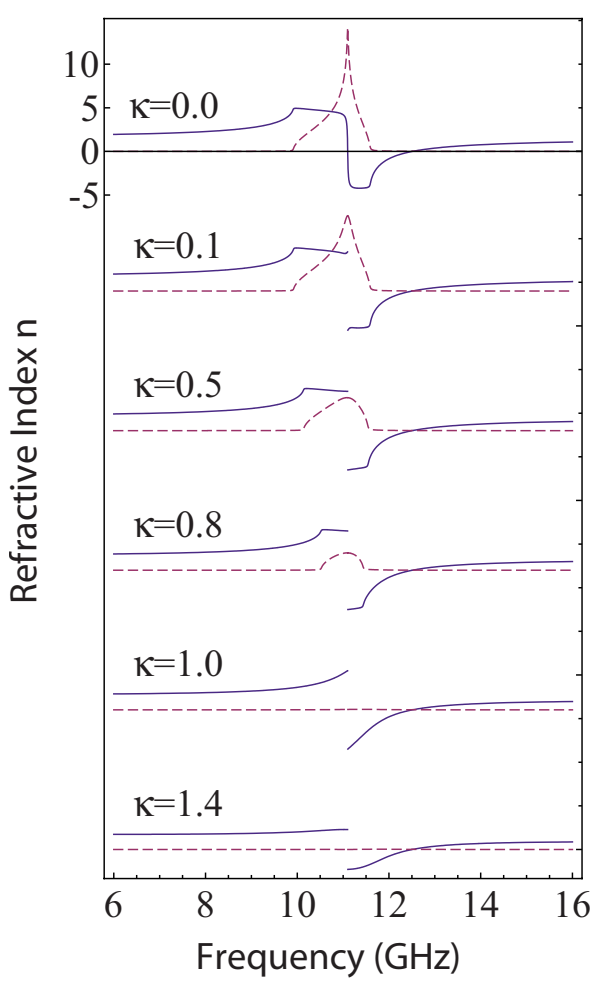

FIG. 5. (Color online) Plots of the real (blue curves) and imaginary (red curves) parts of the effective refractive index corresponding to a unit cell with matched resonant permittivity and permeability. Each plot corresponds to Eq. (63) with a different value of $\kappa$ assumed. In all cases, $\kappa$ is real, having values $0.0,0.1,0.5,0.8,1.0$, and 1.4 , from top to bottom.

electric resonator in Figs. 5 and 6. The resonator properties have been chosen arbitrarily as $f_{0 e}=f_{0 m}=11.1 \mathrm{GHz}, f_{e p}$ $=7.9 \mathrm{GHz}, f_{m p}=5.8 \mathrm{GHz}, \Gamma_{e}=0.01 \mathrm{GHz}, \Gamma_{m}=0.04 \mathrm{GHz}$, $\varepsilon_{b}=1.9$, and $\mu_{b}=1.0$. Figure 5 assumes different values of a real coupling parameter, while Fig. 6 assumes a purely imaginary coupling parameter. It can be seen from the figures that a real coupling parameter tends to suppress the imaginary component of the index, eventually eliminating any apparent band-gap region near the resonance, while an imaginary coupling constant produces what appear to be two resonant regions separated by a section of band gap.

\section{COMPARISONS TO FULL-WAVE SIMULATIONS}

To illustrate the above results, we now consider several examples of metamaterial structures based on the combination of electric and magnetic resonators, comparing their retrieved effective index and Bloch parameters to those predicted analytically using the equations derived in the preceding sections. The $S$-parameter retrieval method we present here makes use of periodic boundary conditions on the surfaces of a single unit cell along the propagation direction, such that a structure effectively infinite in the lateral directions is simulated. The complete set of cell-to-cell interactions is correctly accounted for in the lateral directions (i.e., dipolar and all orders of multipoles). Along the propagation direction, however, the retrieval algorithm used inher-

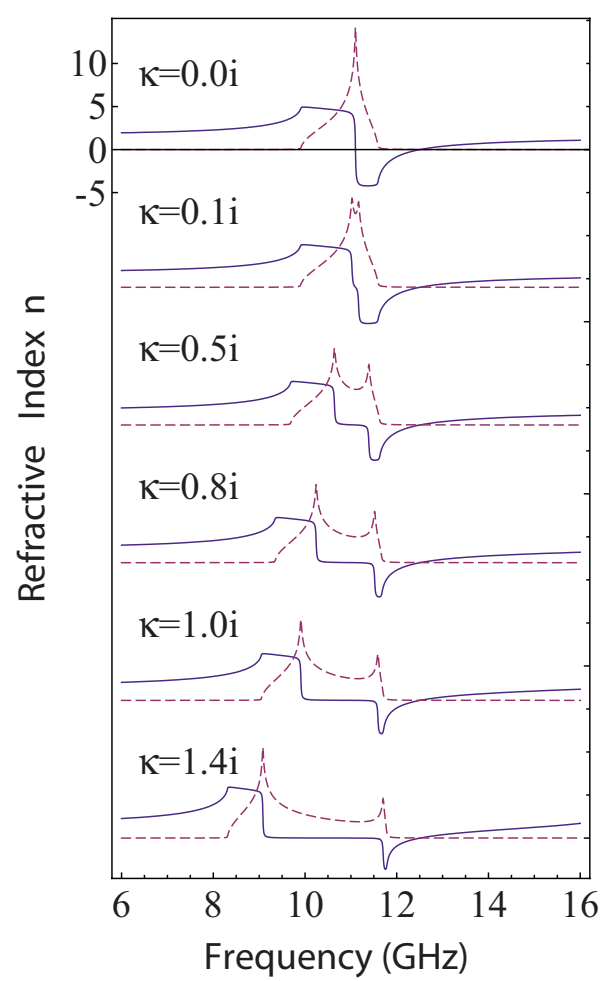

FIG. 6. (Color online) Plots of the real (blue curves) and imaginary (red curves) parts of the refractive index corresponding to a unit cell with matched resonant permittivity and permeability. Each plot corresponds to Eq. (63) with a different value of $\kappa$ assumed. In all cases, $\kappa$ is imaginary, having values $0,0.1 i, 0.5 i, 0.8 i, 1.0 i$, and $1.4 i$ from top to bottom.

ently neglects all cell-to-cell interactions other than the lowest-order Bloch wave, so that any magnetoelectric coupling such as that predicted in Sec. IV cannot be evaluated by this method. Our goals in this section, then, are to compare the analytically predicted forms for the Bloch parameters for single cells to those directly computed from fullwave simulations and to investigate the behavior of inherently magnetoelectric unit cells. Because the matching of magnetic and electric resonators is one approach to forming a negative index medium, we have made an attempt in these examples to match both the resonance frequencies as well as the dispersions of both electric and magnetic resonators to obtain large frequency bandwidths where both the permittivity and the permeability are large in value and both positive or negative.

To facilitate the comparisons, we make use of the standard electric and magnetic metamaterial elements: an electric " $L C$ " resonator (or ELC) [36] and a split-ring resonator (ESRR) [13] for resonant electric and magnetic responses, respectively. In addition, to facilitate a wider variety of coupling conditions, we add to this set the dual structures of a magnetic $L C$ resonator (or MLC) and a magnetic split-ring resonator (MSRR), which have the identical geometries as their electric counterparts but are assumed to have a magnetic conductivity (in this case, a large imaginary permeability value approximates the magnetic conductor). The MLC and MSRR are purely simulation contrivances for illustrative purposes and have no practical utility. All of the numerical 


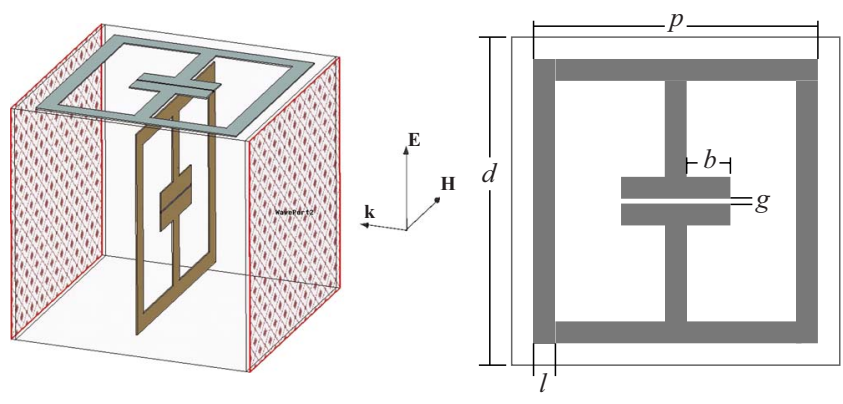

FIG. 7. (Color online) (Left) ELC and MLC structures. The wave is assumed to propagate between the two patterned surfaces, polarized such that the electric field excites the ELC while the magnetic field excites the MLC. (Right) ELC consists of a conducting ring, as shown, with dimensions $d=3 \mathrm{~mm}, p=2.6 \mathrm{~mm}, l$ $=0.2 \mathrm{~mm}, b=0.25 \mathrm{~mm}$, and $g=0.02 \mathrm{~mm}$. The conductor thickness is assumed as $17 \mu \mathrm{m}$. The MLC has precisely the same geometry, but with a magnetic conductivity rather than an electrical conductivity. To lower the resonance frequency, a piece of either dielectric or permeable material is placed within the gaps, with $\varepsilon=6$ for the ELC and $\mu=6$ for the MLC. Periodic boundary conditions are applied on the sides of the unit cell (perpendicular to the propagation direction) to simulate a metamaterial array infinite in the lateral dimensions.

simulations are performed using HFSS (Ansys), a commercial electromagnetic mode solver that is based on the finiteelement method.

For all of the examples in this section, we follow the procedure of computing the scattering $(S)$ parameters for each of the structures utilizing the driven solution in HFSS, with input and output ports defined on two opposing faces of the unit cell. A combination of periodic and electric-magnetic boundary conditions is used both to define the polarization of the wave and to simulate an infinite lattice perpendicular to the direction of propagation [2,3]. From the computed $S$ parameters, the Bloch constitutive parameters can be found by applying a standard retrieval process [2].

The first structure we consider is that of an ELC and MLC pair. The geometry of the resonators is shown in Fig. 7. The elements have been designed such that the electric or magnetic resonances occur near $10 \mathrm{GHz}$, so that the unit cell $(d=3 \mathrm{~mm})$ is nominally a factor of 10 smaller than the freespace wavelength $(3 \mathrm{~cm})$. The ELC and MLC have a single, interior, capacitive gap, which leads to a resonance with relatively lower oscillator strength (i.e., the effective plasma frequencies of these structures will tend to be lower).

The results of retrievals on the unit cell shown in Fig. 7 are shown in Fig. 8. The ELC and the MLC were simulated independently and their Bloch constitutive parameters retrieved [Figs. 8(a)-8(d)]. The ELC alone produces a welldefined electric resonance [Fig. 8(a)], with a corresponding distorted Bloch permeability [Fig. 8(b)] that has become the signature of metamaterial retrievals. The real and imaginary parts of the retrieved Bloch constitutive parameters are shown as the solid blue and red curves, respectively; this labeling will remain consistent throughout all of the illustrative plots. The dashed blue and red curves correspond to the real and imaginary parts of the analytically computed Bloch parameters, found using Eqs. (24) and simple Lorentzians assumed for the oscillators [Eq. (19)] for Figs. 8(a) and 8(b). The parameters used for the Lorentzian fits are summarized in Table I. Note that there is some discrepancy between the simulations and analytical formulas, especially toward higher frequencies; this deviation results because higher-order resonances are not being taken into account in the curve fitting. The fitting performed was simply to obtain a reasonable fit to the curves and was not exhaustive. No attempt was made to include higher-order resonances in the analytical model, which can play a role in determining the line shapes of the Bloch constitutive parameters. A similar retrieval procedure performed for the MLC comparing to Eq. (31) reveals the complementary structure behaves like a magnetic resonator, with properties identical to that of the ELC resonator [Figs. 8(c) and 8(d)].

When the two resonators are combined in the same unit cell, as in Fig. 7, the resulting structure should exhibit a large band of negative index behavior. Were the analytical formulas to describe the simulated structures accurate, we would expect the Lorentzian parameters used to fit the ELC and MLC structures independently would also be correct for the coupled structures. We see from Table I, however, that some adjustments of the oscillator parameters were necessary to obtain the best fits, indicating either that the theory is incomplete or that the value of the coupling parameter $\kappa$ has not been properly chosen. This result is not unexpected, however, since we have not attempted to fully derive the dispersive properties of the coupling parameter $\kappa$, which may not be a simple constant over all frequencies. Note that when the resonators are somewhat detuned, a reasonable fit can be accomplished assuming no coupling [Fig. 8(e)]. Since it is unlikely the coupling parameter is truly zero, the presence of any vestige of the parameter may be introduced in the "detuned" Lorentzian parameter fits, which are found substantially different from the individual ELC or MLC alone.

When the ELC and MLC resonators are combined and tuned (by slightly changing the geometrical features on one or both of the elements), the resulting retrieved refractive index exhibits a substantial region over which the effective refractive index is negative and the imaginary component appears considerably suppressed [Fig. 8(f)]. Were there no interaction between the ELC and the MLC, then a relatively strong band-gap region would be expected, as illustrated by the lighter dashed curves in the figure. However, because of the strong coupling between the resonators, the band-gap region is virtually eliminated and the retrieved index curve can be fit using Eq. (63) with a coupling parameter $\kappa$ near unity (see Table I).

Although the single-gap ELC and MLC provide straightforward structures with which we can explore magnetoelectric coupling, the oscillator strengths of these elements are relatively small. When the effective plasma frequencies for the two resonators are small, it is a difficult task to perfectly match the dispersions to find the narrow region, for example, where the index is negative. By contrast, the dual-gap ELC and MLC elements have considerably stronger oscillator strengths and are consequently much easier to match.

The geometries of the dual-gap ELC and MLC elements are shown in Fig. 9. As for the single-gap resonators, to 

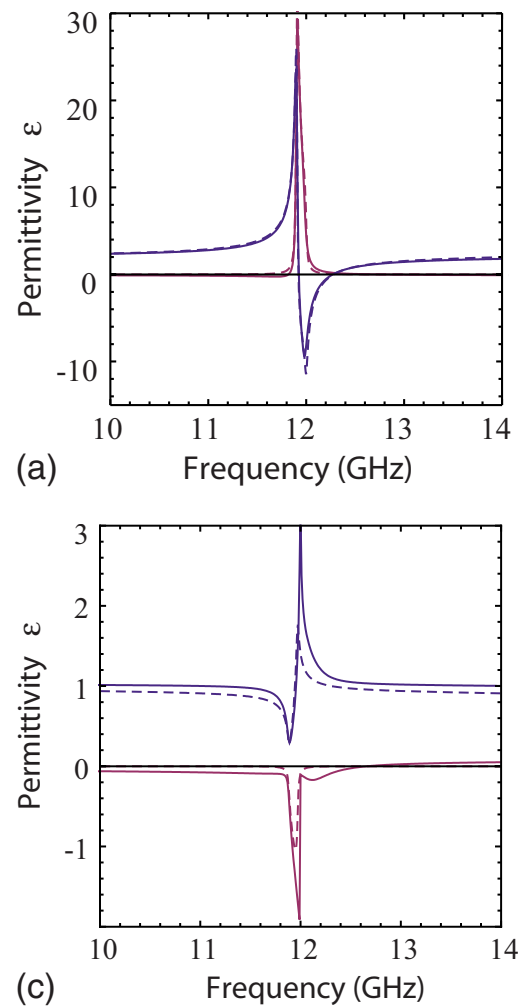

(b)
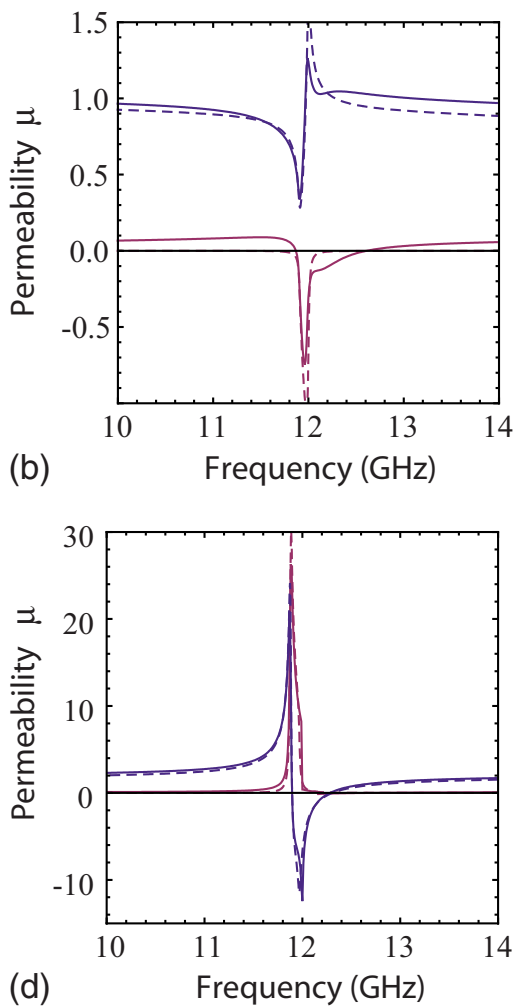

(d)
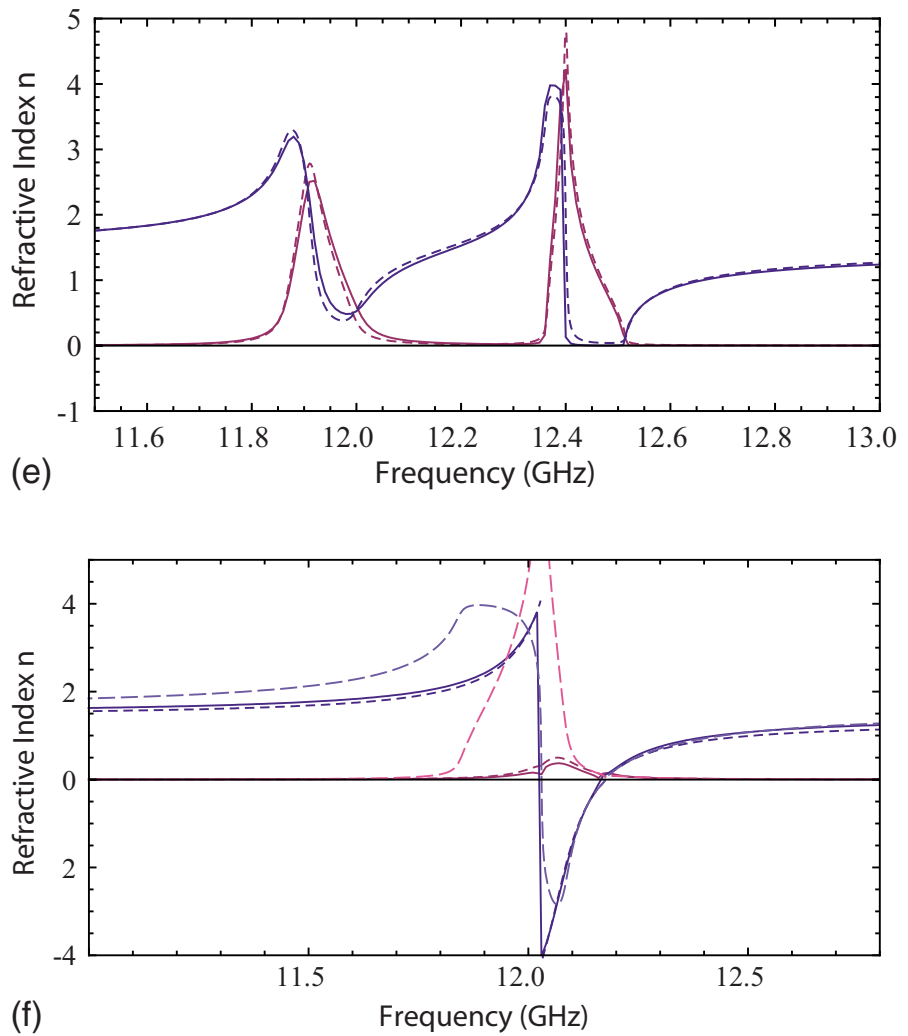

FIG. 8. (Color online) Retrieved Bloch (a) permittivity and (b) permeability for the ELC alone; retrieved Bloch (c) permittivity and (d) permeability for the MLC alone; (e) retrieved index of refraction for the ELC and MLC together, but with resonances slightly detuned; (f) retrieved index of refraction for the ELC and MLC with resonances matched. Solid blue and red lines correspond to the real and imaginary parts of the given parameter retrieved from full-wave simulations, while blue and red dashed lines are fits to the real and imaginary parts of the same parameter using Eq. (24) or (31). The real and imaginary parts of the index (solid blue and red lines) are compared to Eq. (63) (dashed blue and red lines). In ( $\mathrm{g})$, the lighter blue and red dashed curves correspond to Eq. (63) plotted with $\kappa=0$, illustrating the very different behavior expected from coupled vs uncoupled oscillators. confirm the resonance properties of the individual elements, the ELC and MLC are first simulated separately and their effective constitutive parameters recovered. These results are shown in Figs. 10(a)-10(d). The solid lines are the retrieval results from full-wave simulations, while the dashed curves are the analytically computed Bloch parameters taken from
Eq. (24) or (31), which are expected to be valid for electric only or magnetic only response. The Lorentzian parameters are adjusted until the best match with the simulation data is obtained. As before, the agreement is generally quite good, with the deviation toward higher frequencies most likely due to higher-order resonances not being included in the fitting. 
TABLE I. Parameters for the single-gap ELC-MLC lattice.

\begin{tabular}{lcccc}
\hline \hline Parameter & ELC & MLC & ELC-MLC detuned & ELC-MLC tuned \\
\hline$f_{e 0}(\mathrm{GHz})$ & 12.0 & & 12.4 & 12.03 \\
$f_{e p}(\mathrm{GHz})$ & 3.5 & & 2.0 & 2.3 \\
$f_{m 0}(\mathrm{GHz})$ & & 12 & 11.9 & 12.03 \\
$f_{m p}(\mathrm{GHz})$ & & 3.5 & 1.7 & 2.3 \\
$\Gamma_{m}(\mathrm{GHz})$ & & 0.02 & 0.03 & 0.045 \\
$\Gamma_{e}(\mathrm{GHz})$ & 0.02 & & 0.005 & 0.003 \\
$\varepsilon_{b}$ & 1.8 & 1 & 1.4 & 1.5 \\
$\mu_{b}$ & 1 & 1.5 & 1.4 & 1.5 \\
$\kappa$ & 0 & 0 & 0.0 & 0.995 \\
\hline \hline
\end{tabular}

When the resonances are tuned to the same frequency-in this case, by slightly extending the capacitive region on the MLC to $b=0.45$ - the dual gap ELC-MLC unit cell displays the same qualitative coupling behavior as the single-gap structure in Fig. 8(f). As shown in Fig. 10(e), the retrieved index exhibits clear qualitative features suggestive that the coupling between the ELC and MLC is strong. Setting the coupling parameter to zero and utilizing the same Lorentzian parameters in Eq. (63) leads to drastically different behavior, as shown by the second set of dashed curves in Fig. 10(e). In particular, ignoring the coupling leads to a very large electromagnetic band-gap region, characterized by the real part of the refractive index being flattened and accompanied by a large imaginary part. The parameters for the different structures are summarized in Table II.

An ESRR combined with an ELC can also produce a matched magnetoelectric structure, though it is generally more difficult to design the dispersion characteristics of the two resonators to coincide in both magnitude and frequency dependences. The dual-gap ELC, however, has a resonance that is fairly close to that of a similarly sized SRR and the
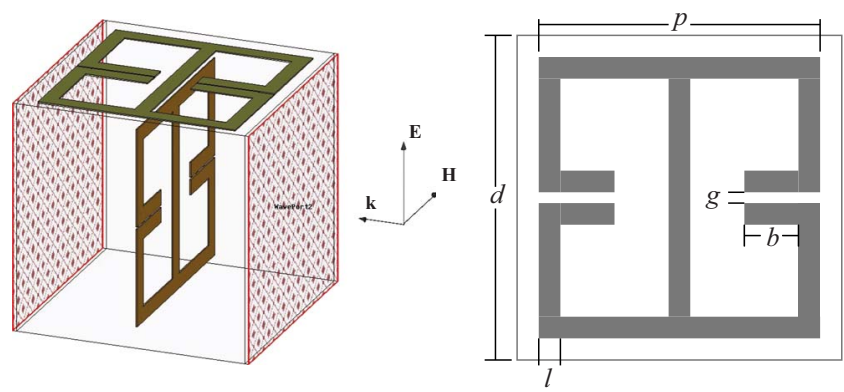

FIG. 9. (Color online) (Left) Dual-gap ELC and MLC structures. The wave is assumed to propagate between the two patterned surfaces, polarized such that the electric field excites the ELC while the magnetic field excites the MLC. (Right) ELC consists of a conducting ring, as shown, with dimensions $d=3 \mathrm{~mm}, p=2.6 \mathrm{~mm}, l$ $=0.2 \mathrm{~mm}, b=0.4 \mathrm{~mm}$, and $g=0.03 \mathrm{~mm}$. The conductor thickness is assumed as $17 \mu \mathrm{m}$. The MLC has precisely the same geometry, but with a magnetic conductivity rather than an electrical conductivity. Also, $b=0.45$ for the MLC, in order to tune the resonances to the same frequency. Periodic boundary conditions are applied on the sides of the unit cell (perpendicular to the propagation direction) to simulate a metamaterial array infinite in the lateral dimensions. combination has been used previously in the experimental demonstration of a negative index metamaterial [37]. We simulate the ELC-ESRR structure here using the geometry shown in Fig. 11, varying the spacing between the ELC and ESRR as an empirical means of adjusting the magnetoelectric coupling. The retrieved Bloch permittivity and permeability for the ELC alone are shown in Figs. 12(a) and 12(b), while similar parameters are shown for the ESRR alone in Figs. 12(c) and 12(d). The figures reveal that the resonances are similar-though not exact—in magnitude and other dispersion characteristics.

When the ELC and ESRR are combined within the same unit cell, similar to Fig. 11, the separation between the ELC and the ESRR can be used to probe varying degrees of coupling. Remarkably, it is possible with this structure to explore the entire range of coupling behavior indicated in Figs. 5 and 6. When the ELC and ESRR are close together-no more than roughly $0.3 \mathrm{~mm}$ apart-the coupling coefficient that matches the retrieved index is found to be purely imaginary, with a magnitude that increases as the elements are moved closer together.

Nearing a separation distance of roughly $0.33 \mathrm{~mm}$, the magnitude of the coupling coefficient appears to become very low, as indicated in Fig. 12(e). The form of the curves in Fig. 12(e) suggests that the magnetoelectric coupling is nearly negligible for this configuration, as evidenced by the apparent band-gap behavior around the resonant frequency of $11 \mathrm{GHz}$. It should be kept in mind, however, that even though there appears to be an absence of magnetoelectric coupling within the unit cell, there will still likely be magnetoelectric coupling between unit cells that is not accounted for in these retrievals. Thus, the retrieval shown in Fig. 12(e) will be unreliable if applied to a sample with a multiple unit cells along the propagation direction since it is Eq. (50) or possibly Eq. (52) that should properly describe the behavior.

After a distance of $0.33 \mathrm{~mm}$, the coupling coefficient becomes purely real and increases as the ELC and ESRR are further separated. In Figs. 12(e) and 12(f), the dashed curves are fit using Eq. (63) with the Lorentzian parameters indicated in Table III. The coupling coefficient, $\kappa$, was assumed constant as a function of frequency.

\section{CONCLUSIONS}

Utilizing a one-dimensional model of plane-wave propagation through a lattice of magnetoelectric sheets, we have investigated a number of issues associated with the development of complex metamaterial structures, in particular those that exhibit magnetoelectric response. In all cases, we have derived approximate analytical expressions to achieve a simpler understanding of the impact of spatial dispersion on the effective constitutive parameters.

The technique of utilizing the simulated $S$ parameters from a single-unit cell for the retrieval of Bloch constitutive parameters is predicated on the assumption that the inherent properties of the unit cell do not change in the presence of the other elements. As recently pointed out [28], this assumption holds for unit cells that exhibit either predominantly electric or predominantly magnetic response, but does not 

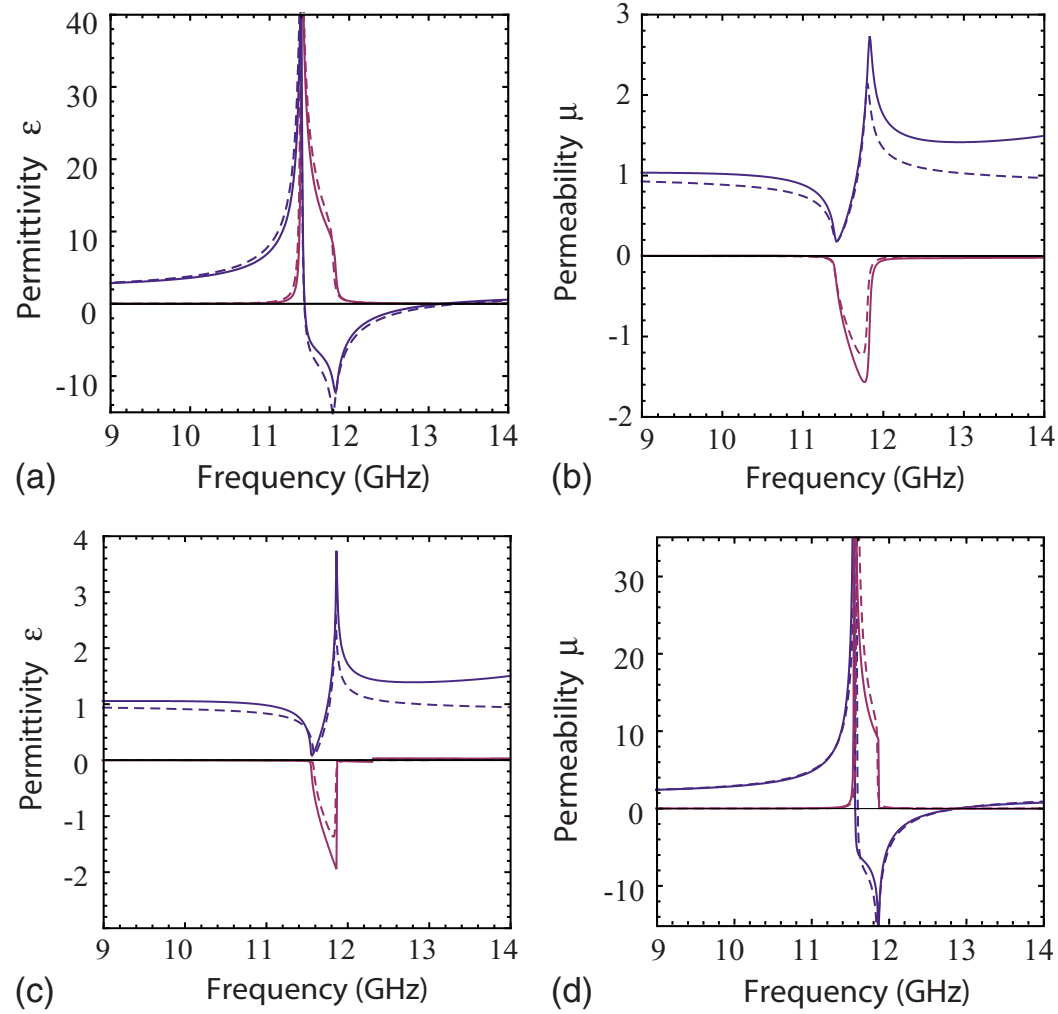

(d)

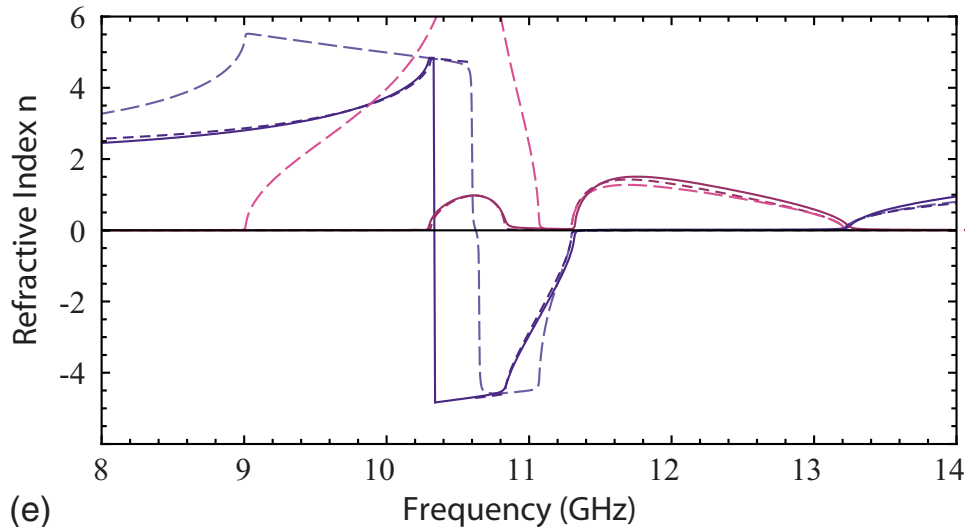

FIG. 10. (Color online) Retrieved Bloch (a) permittivity and (b) permeability for the ELC alone; retrieved Bloch (c) permittivity and (d) permeability for the MLC alone; (e) retrieved index of refraction for the ELC and MLC together with resonances matched, arranged as in Fig. 9. Solid blue and red lines correspond to the real and imaginary parts of the given parameter retrieved from full-wave simulations, while the blue and red dashed lines are fits to the real and imaginary parts of the same parameter using Eq. (24) or (31). The real and imaginary parts of the index (solid blue and red lines) are compared to Eq. (63) (dashed blue and red lines). In (e), the lighter blue and red dashed curves correspond to Eq. (63) plotted with $\kappa=0$, illustrating the very different behavior expected from uncoupled vs coupled oscillators.

hold for unit cells that have simultaneously significant magnetic and electric responses. For such unit cells, a nonlocal interaction is present that results in a more complicated dispersion relation. We have not attempted to verify this expres-

TABLE II. Parameters for the dual-gap ELC-MLC lattice.

\begin{tabular}{lccc}
\hline \hline Parameter & ELC & MLC & ELC-MLC \\
\hline$f_{e 0}(\mathrm{GHz})$ & 11.8 & & 10.65 \\
$f_{e p}(\mathrm{GHz})$ & 7.7 & & 10.2 \\
$f_{m 0}(\mathrm{GHz})$ & & 11.85 & 10.6 \\
$f_{m p}(\mathrm{GHz})$ & & 6.2 & 5.0 \\
$\Gamma_{m}(\mathrm{GHz})$ & & 0.005 & 0.005 \\
$\Gamma_{e}(\mathrm{GHz})$ & 0.04 & & 0.005 \\
$\varepsilon_{b}$ & 1.5 & 1 & 1.7 \\
$\mu_{b}$ & 1 & 1.5 & 1.7 \\
$\kappa$ & 0 & 0 & 0.95 \\
\hline \hline
\end{tabular}

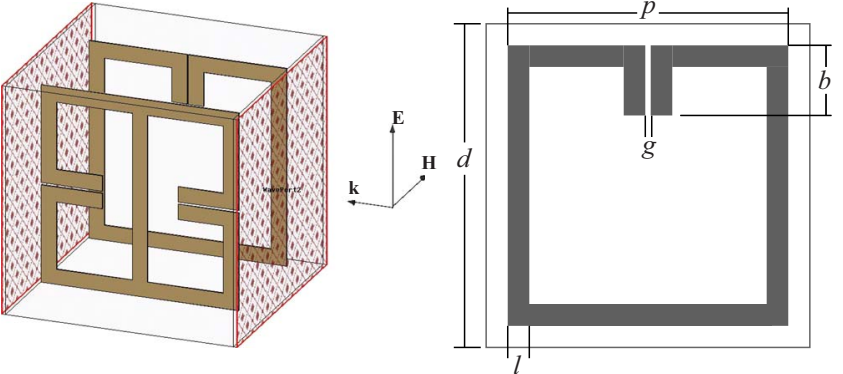

FIG. 11. (Color online) (Left) ELC and ESRR unit cells. (Right) Geometrical parameters for the ELC are identical to those in Fig. 9, while the ESRR has the dimensions $d=3 \mathrm{~mm}$, $p=2.6 \mathrm{~mm}, l=0.2 \mathrm{~mm}, g=0.02 \mathrm{~mm}$, and $b=0.65 \mathrm{~mm}$. Both the ELC and the ESRR are modeled as metals, with an electrical conductivity corresponding to that of copper. The SRR and ELC are placed symmetrically across the midplane of the cell, with a variable distance between them in the direction perpendicular to propagation. 

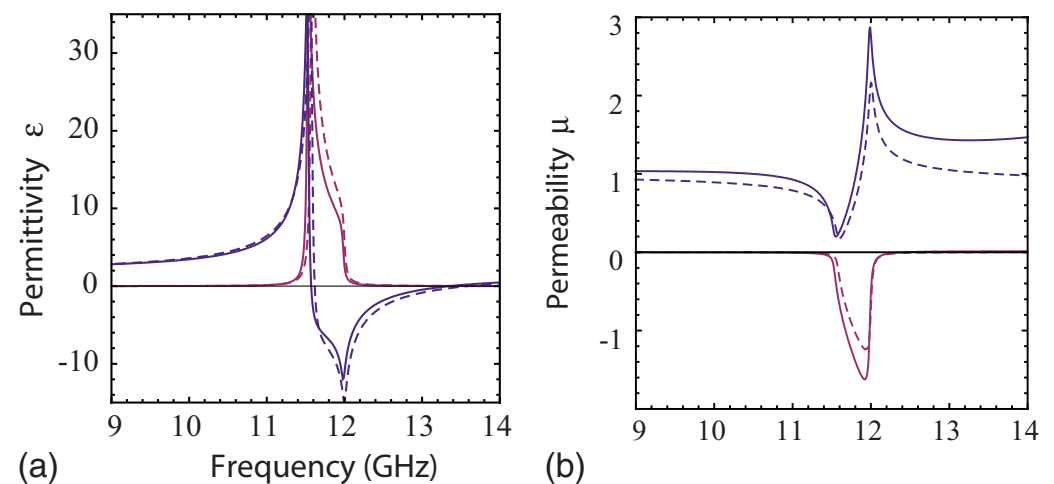

(b)
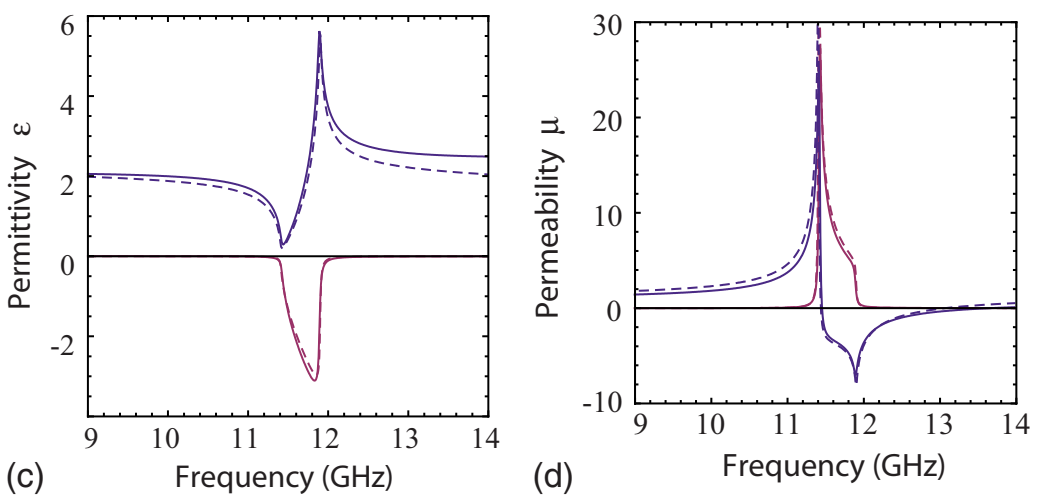

(d)
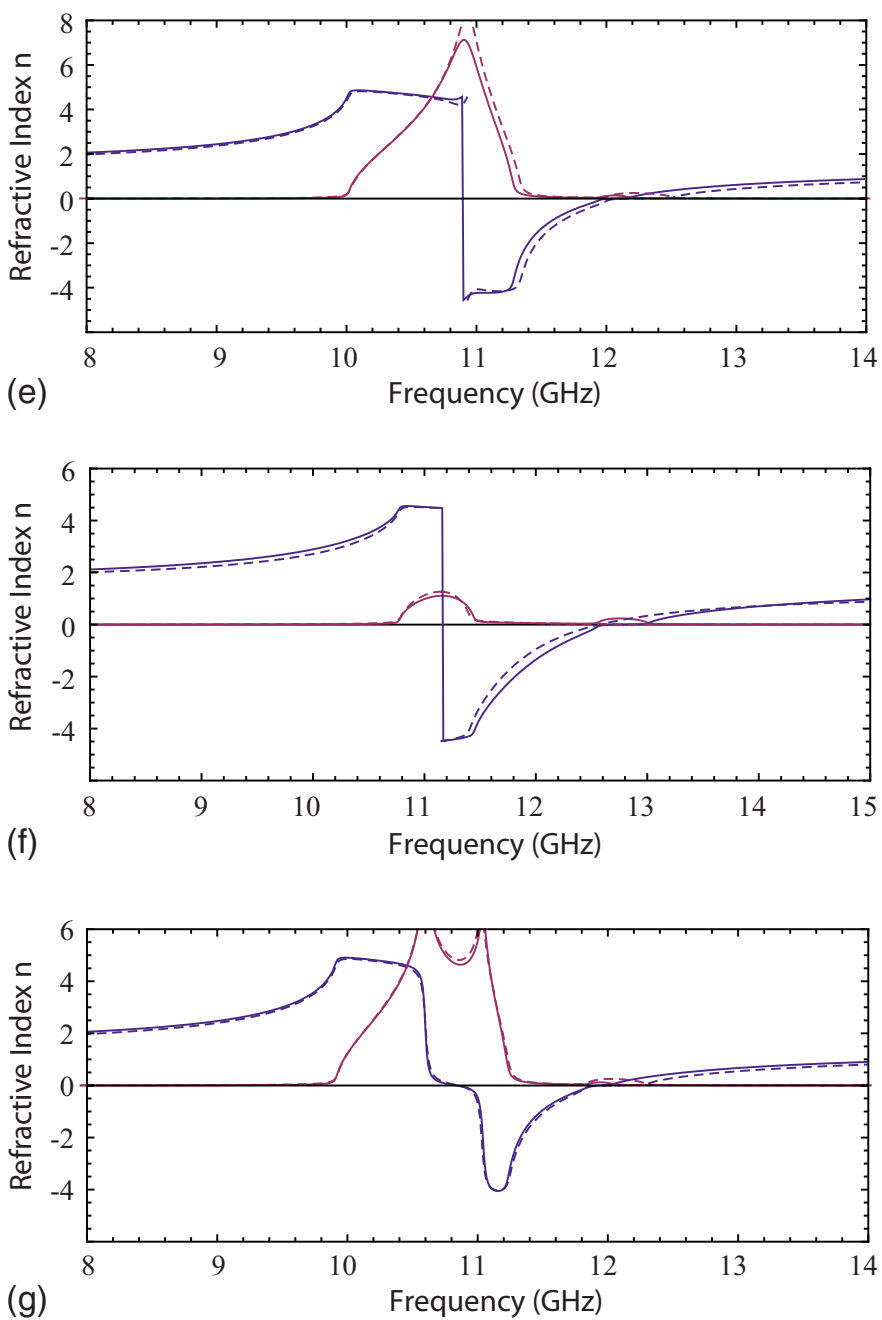

FIG. 12. (Color online) Retrieved Bloch (a) permittivity and (b) permeability for the ELC alone; retrieved Bloch (c) permittivity and (d) permeability for the ESRR alone; (e) retrieved index of refraction for the ELC and ESRR combined, arranged as in Fig. 11, separated by 0.332 $\mathrm{mm}$; (f) retrieved index of refraction for the ELC and ESRR combined, separated by $1.2 \mathrm{~mm}$; $(\mathrm{g})$ retrieved refractive index for the ELC and ESRR combined, separated by $0.3 \mathrm{~mm}$. Solid blue and red lines correspond to the real and imaginary parts of the given parameter retrieved from fullwave simulations, while the blue and red dashed lines are fits to the real and imaginary parts of the same parameter using Eq. (24) or (31). The real and imaginary parts of the index (solid blue and red lines) are compared to Eq. (63) (dashed blue and red lines). The curve fits make use of the parameters shown in Table III. The qualitative features observed in these plots can be compared to those in Figs. 5 and 6. 
TABLE III. Parameters for the ELC-ESRR structure.

\begin{tabular}{lccccc}
\hline \hline & & & ELC-ESRR & ELC-ESRR & ELC-ESRR \\
Parameter & ELC & ESRR & 0.332 mm apart & 1.2 mm apart & 0.3 mm apart \\
\hline$f_{e 0}(\mathrm{GHz})$ & 12.0 & & 10.93 & 11.15 & 10.85 \\
$f_{e p}(\mathrm{GHz})$ & 7.8 & & 6.1 & 7.95 & 5.8 \\
$f_{m 0}(\mathrm{GHz})$ & & 11.9 & 10.93 & 11.15 & 10.85 \\
$f_{m p}(\mathrm{GHz})$ & & 5.4 & 0.1 & 5.85 & 5.8 \\
$\Gamma_{m}(\mathrm{GHz})$ & & 0.04 & 0.04 & 0.04 & 0.04 \\
$\Gamma_{e}(\mathrm{GHz})$ & 0.04 & & 1.5 & 0.04 & 0.04 \\
$\varepsilon_{b}$ & 1.5 & 2.2 & 1 & 1.9 & 1.5 \\
$\mu_{b}$ & 1 & 1 & 0.12 & 0.91 & 1 \\
$\kappa$ & 0 & 0 & & & $0.4 i$ \\
\hline \hline
\end{tabular}

sion numerically here, as doing so would require additional computational methods or the simulation of multiple unit cells, but conclude that retrievals performed on single-unit cells are very likely to yield incorrect results, including for those magnetoelectric cells presented in the previous section.

Empirically, it is found from numerical retrievals on unit cells containing electric and magnetic resonators that the observed refractive index can have very unusual dispersion, not predictable based on a simple model of noninteracting resonances. When we model the unit cell as being composed of two separated sheets, one magnetic and one electric, we find a coupling between them that leads to a description of the unit cell as bianisotropic. Although the model is simple in nature, we find very good agreement between the dispersive forms of the Bloch constitutive parameters predicted by the analytical expressions and those found from numerical retrievals based on full-wave simulations. Depending on the configuration of the resonators, it is possible to find the entire range of apparent coupling conditions predicted by the simple analytical model.

One issue of concern is that the unit cells considered here, being symmetric in the direction of propagation, should strictly not display bianisotropic behavior. We have not attempted to solve this paradox here, but note that the assumption of a thin-sheet model in which the electric and magnetic dipoles are confined to the same plane may not be valid. If one assumes the dipolar response is spread across the unit cell, then effects of spatial dispersion (and the finite wave vector) may break the symmetry and lead to the magnetoelectric coupling that appears to describe the retrieved refractive index. A similar observation was made recently by Fietz and Shvets, who developed a homogenization scheme based on field averaging capable of determining all 36 of the constitutive tensor elements [38].

The agreement between the analytical formulas and the numerical retrievals is persuasive, but does not alone validate the theory. Considerable freedom exists in the curve-fitting process, given the number of parameters in the Lorentzians. Additional fitting parameters can be introduced by consider- ing higher-order resonances of the metamaterial elements. Although the characteristic features of coupling appear to be present in the retrieved index curves, a more direct method of computing the coupling coefficient for a given configuration would be useful.

It should be noted that the simplification in the formulas developed here arises from the fact that the free-space region within the unit cell is much smaller than the wavelength. This regime allows the more involved equations to reduce to remarkably simple expressions that capture most or all of the phenomena associated with metamaterial-retrieved parameters. Although the optical phase advance is not necessarily small across the unit cell in general, by confining the predominant phase advance to occur over a very thin layer-as might be expected for a layer of dipoles - a relatively simple statement of metamaterial response can be encapsulated in formulas such as Eqs. (24), (31), and (63). Though it is not clear that an actual structure comprising elements such as ELCs and ESRRs should adhere to such a simple model, the observed agreement between full-wave simulations and the analytical formulas implies this to be the case.

Our results show that the development of metamaterials with entirely arbitrary response-that is, Bloch permittivity and permeability tensors with arbitrary values for the diagonal elements-will be significantly complicated by unwanted magnetoelectric coupling. Such coupling will need to be mitigated not only in negative index materials, but especially in transformation optical metamaterials [39,40], which are inherently anisotropic and often require both significant electric and magnetic responses.

\section{ACKNOWLEDGMENTS}

The author is grateful for helpful feedback from R. Liu and N. Kundtz, as well as insightful discussions with C. Simovski. This work was supported by the Air Force Office of Scientific Research (USAFOSR) through a Multiple University Research Initiative (Contract No. FA9550-06-1-0279), with partial support from the Air Force Research Laboratory's Space Vehicles Directorate through an SBIR joint with SensorMetriX (Contract No. FA9453-09-C-0023). 
[1] D. R. Smith, D. C. Vier, N. Kroll, and S. Schultz, Appl. Phys. Lett. 77, 2246 (2000).

[2] D. R. Smith, S. Schultz, P. Markoš, and C. M. Soukoulis, Phys. Rev. B 65, 195104 (2002).

[3] D. R. Smith, D. C. Vier, Th. Koschny, and C. M. Soukoulis, Phys. Rev. E 71, 036617 (2005).

[4] D. R. Smith and J. B. Pendry, J. Opt. Soc. Am. B 23, 391 (2006).

[5] R. Liu, T. J. Cui, D. Huang, B. Zhao, and D. R. Smith, Phys. Rev. E 76, 026606 (2007).

[6] C. R. Simovski and S. A. Tretyakov, Phys. Rev. B 75, 195111 (2007).

[7] L. D. Landau, E. M. Lifshitz, and L. P. Pitaevskii, Electrodynamics of Continuous Media, 2nd ed. (Butterworth-Heinenann, Oxford, 1984).

[8] V. M. Shalaev, Phys. Rep. 272, 61 (1996).

[9] D. J. Bergman, Phys. Rep. 43(9), 377 (1978).

[10] T. C. Choy, Effective Medium Theory (Clarendon, Oxford, 1999).

[11] A. Sihvola, Electromagnetic Mixing Formulae and Applications, IEE Electromagnetic Wave Series Vol. 47 (The Institute of Electrical Engineers, London, 1999).

[12] J. B. Pendry, A. J. Holden, W. J. Stewart, and I. Youngs, Phys. Rev. Lett. 76, 4773 (1996).

[13] J. B. Pendry, A. J. Holden, D. J. Robbins, and W. J. Stewart, IEEE Trans. Microwave Theory Tech. 47, 2075 (1999).

[14] D. R. Smith, W. Padilla, D. C. Vier, S. C. Nemat-Nasser, and S. Schultz, Phys. Rev. Lett. 84, 4184 (2000).

[15] M. Bayindir, K. Aydin, E. Ozbay, P. Markos, and C. M. Soukoulis, Appl. Phys. Lett. 81, 120 (2002).

[16] C. G. Parazzoli, R. B. Greegor, K. Li, B. E. C. Koltenbah, and M. Tanielian, Phys. Rev. Lett. 90, 107401 (2003).

[17] P. F. Loschialpo, D. L. Smith, D. W. Forester, F. J. Rachford, and J. Schelleng, Phys. Rev. E 67, 025602 (2003).

[18] A. A. Houck, J. B. Brock, and I. L. Chuang, Phys. Rev. Lett. 90, 137401 (2003).

[19] R. W. Ziolkowski, IEEE Trans. Antennas Propag. 51, 1516 (2003).

[20] F. Falcone, T. Lopetegi, M. A. G. Laso, J. D. Baena, J. Bonache, M. Beruete, R. Marques, F. Martin, and M. Sorolla, Phys. Rev. Lett. 93, 197401 (2004).
[21] D. R. Smith, J. J. Mock, A. F. Starr, and D. Schurig, Phys. Rev. E 71, 036609 (2005).

[22] R. B. Greegor, C. G. Parazzoli, J. A. Nielsen, M. A. Thompson, M. H. Tanielian, and D. R. Smith, Appl. Phys. Lett. 87, 091114 (2005).

[23] T. Driscoll, D. N. Basov, A. F. Starr, P. M. Rye, S. NematNasser, D. Schurig, and D. R. Smith, Appl. Phys. Lett. 88, 081101 (2006).

[24] D. Schurig, J. J. Mock, B. J. Justice, S. A. Cummer, J. B. Pendry, A. F. Starr, and D. R. Smith, Science 314, 977 (2006).

[25] R. Liu, C. Ji, J. J. Mock, J. Y. Chin, T. J. Cui, and D. R. Smith, Science 323, 366 (2009).

[26] T. Koschny, P. Markos, D. R. Smith, and C. M. Soukoulis, Phys. Rev. E 68, 065602 (2003).

[27] T. Koschny, P. Markos, E. N. Economou, D. R. Smith, D. C. Vier, and C. M. Soukoulis, Phys. Rev. B 71, 245105 (2005).

[28] C. R. Simovski, Metamaterials 1, 62 (2007).

[29] R. E. Collin, Field Theory of Guided Waves, 2nd ed. (Wiley, New York, 1998).

[30] P. A. Belov and C. R. Simovski, Phys. Rev. E 72, 026615 (2005).

[31] S. A. Tretyakov, Analytical Modeling in Applied Electromagnetics (Artec House, Norwood, MA, 2003).

[32] P. Markos and C. M. Soukoulis, Wave Propagation: From Electrons to Photonic Crystals and Left-Handed Materials (Princeton University Press, Princeton, NJ, 2008).

[33] C. R. Simovski and S. He, Phys. Lett. A 311, 254 (2003).

[34] R. Marques, F. Medina, and R. Rafii-El-Idrissi, Phys. Rev. B 65, 144440 (2002).

[35] V. Dmitriev, Electron. Lett. 34, 743 (1998).

[36] D. R. Schurig, J. J. Mock, and D. R. Smith, Appl. Phys. Lett. 88, 041109 (2006).

[37] R. Liu, A. Degiron, J. J. Mock, and D. R. Smith, Appl. Phys. Lett. 90, 263504 (2007).

[38] C. Fietz and G. Shvets, in Metamaterials: Fundamentals and Applications II, edited by M. A. Noginov, N. I. Zheludev, A. D. Boardman, and N. Engheta, Proceedings of SPIE Vol. 7392 (SPIE, Bellingham, WA, 2009), p. 19.

[39] J. B. Pendry, D. Schurig, and D. R. Smith, Science 312, 1780 (2006).

[40] U. Leonhardt, Science 312, 1777 (2006). 